\title{
A retrospective comparison for prediction of optimal length of right subclavian vein catheterization in infants: landmark-based estimation vs. linear regression model
}

\section{Chahyun Oh ${ }^{1,2}$, Boohwi Hong ${ }^{1,2}$, Yumin Jo, ${ }^{1,2}$, Woosuk Chung ${ }^{1,2}$ Hoseop Kim ${ }^{1}$, Suyeon Shin ${ }^{1}$, Ah Young Choi ${ }^{3}$, Chaeseong Lim ${ }^{1,2}$, Youngkwon Ko ${ }^{1,2}$, Yoon-Hee Kim ${ }^{1,2}$, and Sun Yeul Lee ${ }^{1,2}$}

${ }^{1}$ Department of Anesthesiology and Pain Medicine, Chungnam National University Hospital, ${ }^{2}$ Department of Anesthesiology and Pain Medicine, College of Medicine, Chungnam National University, ${ }^{3}$ Department of Pediatrics, Chungnam National University Hospital, Daejeon, Korea
Received March 8, 2021

Revised June 18, 2021

Accepted June 21, 2021

\section{Corresponding author}

Sun Yeul Lee, M.D., Ph.D.

Department of Anesthesiology and

Pain Medicine, Chungnam National

University Hospital, 282 Munhwa-ro,

Jung-gu, Daejeon 35015, Korea

Tel: 82-42-280-7840

Fax: 82-42-280-7968

E-mail: neoquack@gmail.com

Chahyun Oh and Boohwi Hong contributed equally to this study.
Background: The optimal insertion length for right subclavian vein catheterization in infants has not been determined. This study retrospectively compared landmark-based and linear regression model-based estimation of optimal insertion length for right subclavian vein catheterization in pediatric patients of corrected age $<1$ year.

Methods: Fifty catheterizations of the right subclavian vein were analyzed. The landmark related distances were: from the needle insertion point $(\mathrm{I})$ to the tip of the sternal head of the right clavicle $(A)$ and from $A$ to the midpoint $(B)$ of the perpendicular line drawn from the sternal head of the right clavicle to the line connecting the nipples. The optimal length of insertion was retrospectively determined by reviewing post-procedural chest radiographs. Estimates using a landmark-based equation (IA + AB - intercept) and a linear regression model were compared with the optimal length of insertion.

Results: A landmark-based equation was determined as IA + AB - 5. The mean difference between the landmark-based estimate and the optimal insertion length was $1.0 \mathrm{~mm}$ (95\% limits of agreement -18.2 to $20.3 \mathrm{~mm}$ ). The mean difference between the linear regression model (26.681 - $4.014 \times$ weight $+0.576 \times \mathrm{IA}+0.537 \times \mathrm{AB}-0.482 \times$ postmenstrual age $)$ and the optimal insertion length was $0 \mathrm{~mm}$ (95\% limits of agreement -16.7 to $16.7 \mathrm{~mm}$ ). The difference between the estimates using these two methods was not significant.

Conclusions: A simple landmark-based equation may be useful for estimating optimal insertion length in pediatric patients of corrected age $<1$ year undergoing right subclavian vein catheterization.

Keywords: Anatomic landmarks; Central venous catheterization; Infant; Pediatrics; Subclavian vein.

This is an Open Access article distributed under the terms of the Creative Commons Attribution Non-Commercial License (http://creativecommons.org/licenses/by-nc/4.0) which permits unrestricted non-commercial use, distribution, and reproduction in any medium, provided the original work is properly cited.

Copyright (C) the Korean Society of Anesthesiologists, 2021 


\section{INTRODUCTION}

Although there are several essential indications for central vein catheterization in small pediatric patients [1], catheterization is not easily achieved in these patients because of their small size and because the vessels can easily collapse. The subclavian vein, which runs underneath the clavicle and lies deeper than the internal jugular vein, is less collapsible and associated with a lower infection rate [2]. Subclavian vein catheterization can be performed safely and efficiently under real-time ultrasound guidance [3].

Central venous catheterization has been successfully performed in pediatric patients using a classical infraclavicular approach with ultrasound scanning at the supraclavicular level $[4,5]$. However, the optimal length of catheter insertion in pediatric patients undergoing subclavian venous catheterization has not been determined. Most methods suggested to date utilize an approach through the internal jugular vein or are less intuitive [6-9].

A simple landmark-based equation has been suggested for internal jugular vein catheterization [8]. This method allows a catheter tip to be positioned at the optimal target on chest radiographs $[8,10]$. This method does not require pre-procedural assessment of demographic or radiologic characteristics and the landmark-based distances can be easily measured during the procedure. However, this method has not yet been validated for subclavian catheterization. This study therefore retrospectively assessed the feasibility of the simple landmark-based equation in pediatric patients of corrected age $<1$ year.

\section{MATERIALS AND METHODS}

\section{Study design and population}

The protocol of this retrospective study was approved by the Institutional Review Board of Chungnam National University Hospital (CNUH 2020-04-12), which waived the requirement for informed consent because of the retrospective nature of the study. The medical records and post-procedural notes recorded by the physician who performed the central catheterization via the right subclavian vein between 2016 and 2019 were reviewed. Patients were excluded if they were of corrected age $>1$ year. Corrected age was calculated by subtracting the number of weeks born before 40 weeks of gestation from the chronological age [11]. Postmenstrual age was calculated by adding the gestational age and chronolog- ical age; thus, a 1-year-old infant born at full-term (gestational age 40 weeks) would have a postmenstrual age of 92 weeks.

\section{Infraclavicular approach to the subclavian vein}

All procedures were performed as described previously $[5,8]$. A linear ultrasound probe was placed at the supraclavicular level with the clavicle at the center of the view. The probe was then directed slightly inferolateral to the superomedial direction. Using an ultrasound-guided in-plane technique, the subclavian vein was punctured with an introducer needle via the infraclavicular route (Fig. 1B). A guidewire was inserted through the needle and the internal jugular vein was scanned to rule out cephalad insertion. The distances between the landmark points were measured during the procedure with a sterile ruler. These measurements included the distances from the needle insertion point (I) to the tip of the sternal head of the right clavicle (A) and from point A to the midpoint (B) of the perpendicular line drawn from the sternal head of the right clavicle to the line connecting the nipples (Fig. 1A). The initial insertion length was determined by adding the distances from I to A and from $\mathrm{A}$ to $B$ and fixed after adjusting the length within a $1 \mathrm{~cm}$ range by considering the occurrence of an arrhythmia, resistance during aspiration, and the placement of an attachment clip.

\section{Data acquisition}

Landmark-based estimation has been used for catheterization in our center since 2016, and the measurements were included in post-procedural notes. Gestational age (weeks), chronological age (weeks), postmenstrual age (weeks), sex, birth weight, height, weight, length of the inserted catheter, and landmark related measurements (I to A [IA] and A to B [AB]) were recorded.

Anteroposterior chest radiographs were obtained after the procedure and reviewed by one author. The vertical distance from the carina to the CVC tip was measured using the Picture Archiving and Communicating System (PACS) (Maroview, Marosis, Korea). The optimal insertion length, defined as the distance at which the catheter tip was positioned at the level of the carina, was calculated as the actual inserted length \pm the vertical distance between the catheter tip and the carina (Fig. 2). Correction for vertical distance was based on the consideration that the catheter usually runs vertically from the superior vena cava to the right atrium. 

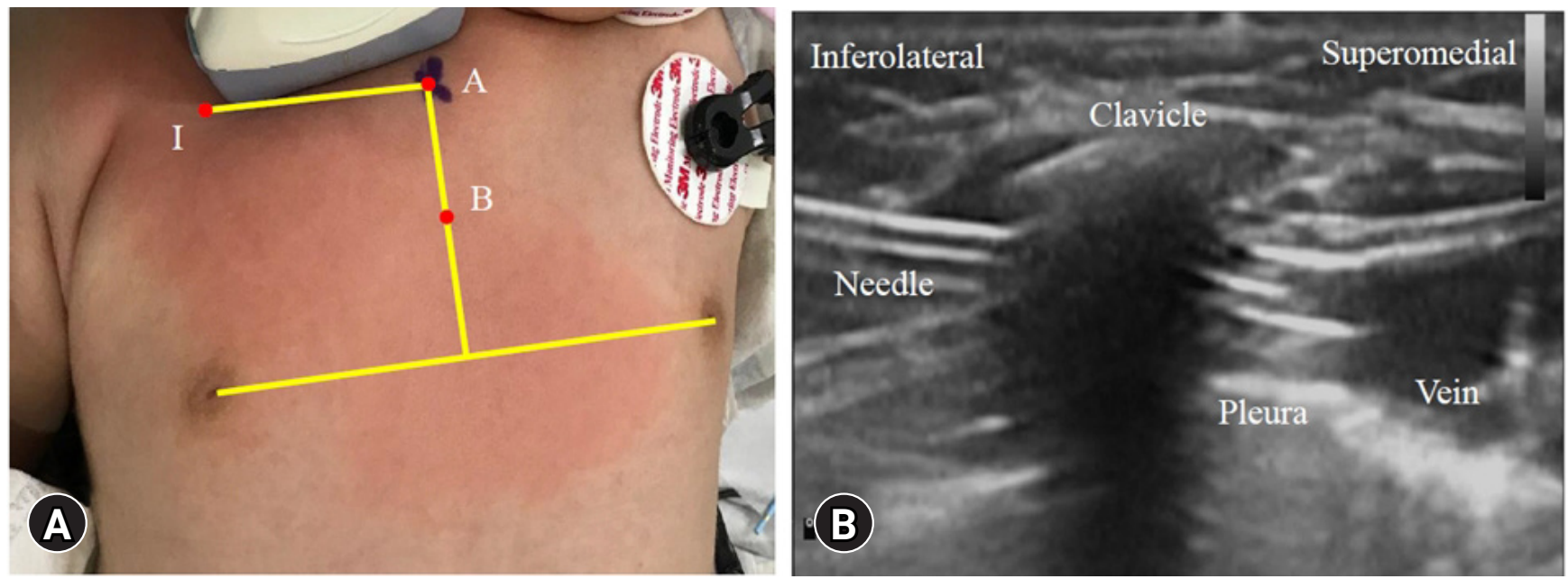

Fig. 1. Landmark points used in landmark-based models $(A)$ and ultrasound image taken during introducer needle insertion via the infraclavicular approach using an in-plane technique (B). (A) I: insertion point of the needle, A: tip of the sternal head of the right clavicle, B: midpoint of the perpendicular line drawn from the sternal head of the right clavicle to the line connecting the nipples. (B) A linear ultrasound probe was placed at the supraclavicular level with the clavicle at the center of the view. The probe was directed slightly inferolateral to superomedial direction. The vein indicated in the figure represents subclavian vein joining with the internal jugular vein.

\section{Derivation of the estimation method}

Landmark-based estimations (IA + AB) were compared with determined optimal insertion lengths (actual inserted length \pm vertical distance). These estimates were subsequently adjusted by determining the optimal intercept that minimizes the mean difference between the determined and estimated optimal insertion lengths.

To determine whether a more precise estimate can be derived from the data of the present study, a regression model was derived by a multiple linear regression analysis that included patient characteristics such as sex, height, weight, birth weight, chronological age, gestational age, postmenstrual age, IA, and AB. Variables with $\mathrm{P}$ values $<0.1$ on univariate analysis were included in a multivariate analysis. When multicollinearity was detected (based on a variance inflation factor $>10$ ), the more clinically meaningful or practical variable was selected. Finally, a model with the lowest Mallow's $\mathrm{C}_{\mathrm{p}}$ was chosen using a best subset selection approach.

\section{Statistical analysis}

The sample size was based on the available data from January 2016 to December 2019. No statistical power calculation was performed before the study. Statistical analysis was performed using R software version 4.0.3 (R Project for Statistical Computing, Austria). Continuous variables are presented as

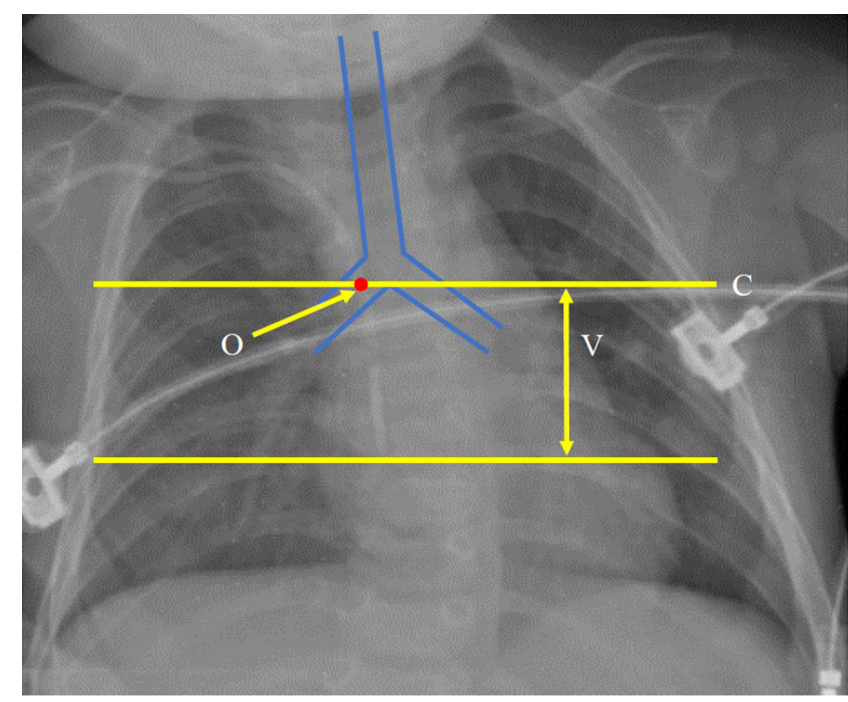

Fig. 2. Determination of the optimal insertion length on chest radiograph. The trachea and main bronchus are outlined by a blue solid line. The optimal insertion length was determined by subtracting the vertical distance $(\mathrm{V})$ between the tip and the carina $(\mathrm{C})$ from the actual inserted length. $\mathrm{C}$ : level of the carina, $\mathrm{V}$ : vertical distance between the catheter tip and the carina, 0 : optimal position of the catheter tip.

mean \pm standard deviation (SD), with $95 \%$ confidence interval (CI), or medians and interquartile ranges (IQR) after testing for normal distribution using the Shapiro-Wilk test. The determined and estimated optimal insertion lengths were compared using the Bland-Altman method, which describes agreement between two quantitative measurements [12]. 
The mean difference (estimated - determined optimal length of insertion) and the $95 \%$ limits of agreement $( \pm 1.96$ SD of the difference) were calculated. A clinically acceptable limit was not defined a priori. The estimates from the landmark-based equation and the linear regression model were compared using paired $t$-tests. Two-tailed $\mathrm{P}$ values $<0.05$ were considered statistically significant.

\section{RESULTS}

Of the 51 right subclavian catheterizations performed in 42 pediatric patients, one catheterization was excluded because of patient age. Thus, this analysis included 50 catheterizations in 41 patients (Fig. 3); their demographic and clinical characteristics are summarized in Table 1.

The difference between the optimal insertion length and the initial landmark-based estimation $(\mathrm{IA}+\mathrm{AB})$ was $6.04 \pm$ $9.81 \mathrm{~mm}$. For practical reasons, $5 \mathrm{~mm}$ was subtracted from each estimate $\left(R^{2}=0.361\right)$. The comparison between the determined optimal insertion length and the adjusted result $(\mathrm{IA}+\mathrm{AB}-5)$ is shown in Fig. 4. The mean difference was 1.04 $\mathrm{mm}$, with the $95 \%$ limits of agreement being $-18.18 \mathrm{~mm}$ and $20.26 \mathrm{~mm}$.

Results of univariate and multivariate analyses are presented in Table 2. In the final model, postmenstrual age (weeks), weight $(\mathrm{kg}), \mathrm{IA}(\mathrm{mm})$, and $\mathrm{AB}(\mathrm{mm})$ were selected, with predicted length calculated as $26.681-4.014 \times$ weight $+0.576 \times \mathrm{IA}+0.537 \times \mathrm{AB}-0.482 \times$ postmenstrual age $(\mathrm{P}<$ 0.001 , adjusted $\left.R^{2}=0.396\right)$. A comparison between the determined optimal insertion length and the estimates using the final model is shown in Fig. 5. The mean difference was 0 $\mathrm{mm}$ and the $95 \%$ limits of agreement were $-16.66 \mathrm{~mm}$ and $16.66 \mathrm{~mm}$.

The difference between the estimates using the landmark-based equation and the linear regression model was not significant (mean difference $-1.04 \mathrm{~mm}, 95 \% \mathrm{CI}-2.43$ to 0.35 $\mathrm{mm})$.

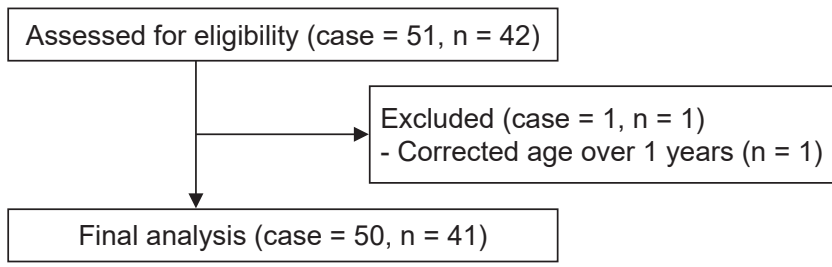

Fig. 3. Flow diagram of patient selection.

\section{DISCUSSION}

This study compared the ability of two models, a landmark-based model and a linear regression model, to predict optimal insertion length during right subclavian vein catheterization in pediatric patients of corrected age $<1$ year. Based on Bland-Altman analysis, the linear regression model was slightly more accurate than the simple landmark-based method. For practical reasons, however, we suggest that a simple landmark-based method be used rather than an estimation based on complicated calculations. The results of the present study indicate that, with a slight adjustment, clinicians can estimate the optimal insertion length by simple measurement and arithmetic. This simplicity may enhance the applicability of the method.

Several other methods have been suggested to determine the optimal insertion length of central catheters [7,13-15]. Most of these methods, however, are based on demographic data, may not be intuitive, and/or require complicated intraoperative calculations. Additionally, these methods may not include considerations of inevitable variations due to actual puncture sites. Based on the results of our multivariate analysis, the actual puncture site (IA) was the most important variable for estimating optimal insertion length. In this context, we considered the method suggested by Na et al. [8] (a

Table 1. Demographic and Clinical Characteristics of the Study Subjects and Catheterizations

\begin{tabular}{lc}
\hline \multicolumn{1}{c}{ Variable } & Value \\
\hline Total catheterizations $(\mathrm{n})$ & 50 \\
Gestational age (wk) & $37.0(32.0,38.0)$ \\
Chronological age (wk) & $13.5(4.0,25.0)$ \\
Postmenstrual age (wk) & $47.0(41.0,56.0)$ \\
Birth weight (g) & $2,550.0(1,870.0,3,100.0)$ \\
Weight (kg) & $4.1(3.0,5.3)$ \\
Height (cm) & $53.5(48.5,59.6)$ \\
Sex (M/F) & $25 / 25$ \\
IA (mm) & $40.0(30.0,40.0)$ \\
AB (mm) & $25.0(20.0,30.0)$ \\
IA + AB (mm) & $59.9 \pm 10.5$ \\
Vertical distance* (mm) & $11.3 \pm 12.5$ \\
Optimal length ${ }^{\dagger}$ (determined, mm) & $53.9 \pm 11.4$ \\
\hline
\end{tabular}

All catheterizations were considered independent events. Values are expressed as number only, median (1Q, 3Q), or mean \pm SD. IA: distance from the insertion point (I) to the tip of the sternal head of the right clavicle $(A), A B$ : distance from point $A$ to the midpoint $(B)$ of the perpendicular line drawn from the sternal head of the right clavicle to the line connecting the nipples. *Vertical distance between the catheter tip and the carina. ${ }^{\dagger}$ Actual inserted length \pm vertical distance. 
method that includes the insertion point as an aspect of estimation) practical and also applicable to subclavian vein catheterization. To prevent inconsistencies between predetermined and actual puncture sites, measurements based on landmark points were performed using a sterile ruler immediately after the guidewire was introduced into the vascular lumen.

The infraclavicular approach to the subclavian vein has

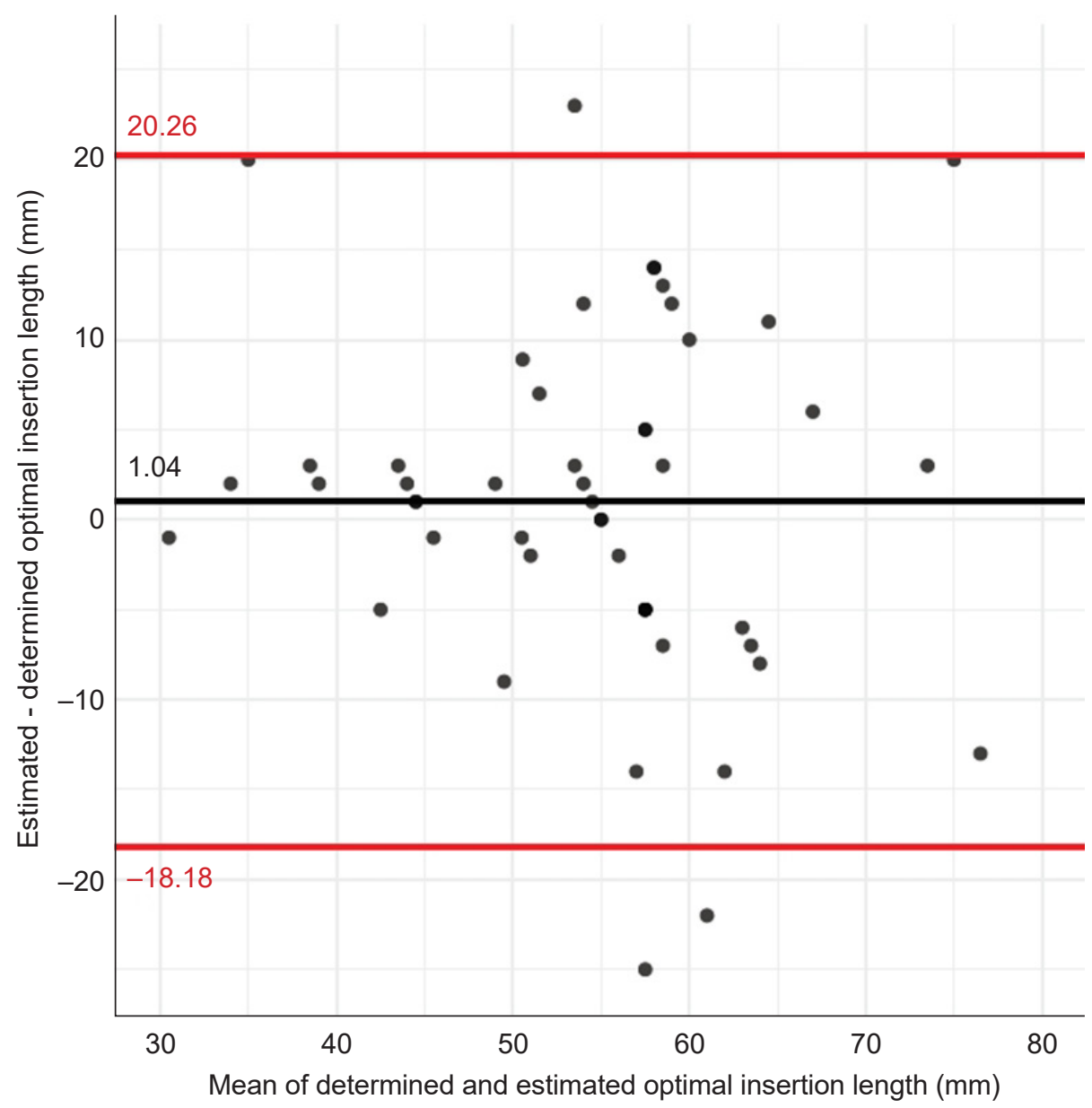

Fig. 4. Bland-Altman plot of determined and estimated optimal insertion lengths $(I A+A B-5)$. IA: distance from the insertion point $(I)$ to the tip of the sternal head of the right clavicle $(A), A B$ : distance from point $A$ to the midpoint $(B)$ of the perpendicular line drawn from the sternal head of the right clavicle to the line connecting the nipples.

Table 2. Univariate and Multivariate Analysis of Factors Associated with Optimal Insertion Depth in Right Subclavian Vein Catheterization

\begin{tabular}{|c|c|c|c|c|c|c|c|}
\hline \multirow{2}{*}{ Variable } & \multicolumn{3}{|c|}{ Univariate } & \multicolumn{3}{|c|}{ Multivariate } & \multirow{2}{*}{ Importance (\% } \\
\hline & Estimate & $95 \% \mathrm{Cl}$ & $P$ value & Estimate & $95 \% \mathrm{Cl}$ & $P$ value & \\
\hline Gestational age (wk) & 0.506 & -0.197 to 1.208 & 0.154 & NA & NA & NA & NA \\
\hline Chronological age $(w k) *$ & 0.269 & 0.027 to 0.511 & 0.030 & NA & NA & NA & NA \\
\hline Postmenstrual age (wk)* & 0.370 & 0.125 to 0.616 & 0.004 & -0.531 & -1.162 to 0.100 & 0.097 & 9.57 \\
\hline Birth weight (g) & 0.004 & 0.001 to 0.007 & 0.022 & 0.001 & -0.003 to 0.004 & 0.701 & 9.33 \\
\hline Weight (kg) & 2.928 & 1.459 to 4.398 & $<0.001$ & 2.814 & -1.079 to 6.707 & 0.152 & 16.54 \\
\hline Height (cm) & 0.663 & 0.346 to 0.980 & $<0.001$ & 0.366 & -0.440 to 1.171 & 0.365 & 16.08 \\
\hline Sex, male & 2.044 & -4.484 to 8.572 & 0.532 & NA & NA & NA & NA \\
\hline $\mathrm{IA}(\mathrm{mm})$ & 0.733 & 0.388 to 1.078 & $<0.001$ & 0.534 & 0.167 to 0.901 & 0.005 & 33.89 \\
\hline $\mathrm{AB}(\mathrm{mm})$ & 0.752 & 0.193 to 1.311 & 0.009 & 0.486 & -0.034 to 1.006 & 0.066 & 14.59 \\
\hline
\end{tabular}

IA: distance from the insertion point (I) to the tip of the sternal head of the right clavicle (A), AB: distance from point $A$ to the midpoint (B) of the perpendicular line drawn from the sternal head of the right clavicle to the line connecting the nipples, Cl: confidence interval, NA: not available. *Postmenstrual age was selected instead of chronological age for the multivariate analysis because of their multicollinearity. 
several advantages over internal jugular vein catheterization in infants [4]. During cannulation, the internal jugular vein tends to collapse easily in response to pressure from the probe or needle. Moreover, multiple attempts to perform internal jugular vein cannulation can result in hematoma around the blood vessels. In contrast, the subclavian vein is less prone to collapse during cannulation because it is suspended within the soft tissue underlying the clavicle [16]. Also, the infraclavicular approach allows direct visualization of needle advancement, reducing the risk of complications and improving the rate of successful placement. An ordinary linear probe, instead of a hockey-stick shaped probe, was shown to be successful in the infraclavicular approach for infants [5]. However, a skilled in-plane technique is required to prevent serious complications in these small infants.

The optimal position of the central catheter tip remains unclear [17]. Vessel injury and thrombosis may be avoided and proper functioning of the catheter maintained by positioning the catheter tip in the right atrium [18]. However, various problems are associated with deep catheter inser- tion, including arrhythmia, endocardial injury, and even cardiac perforation and tamponade [19-21]. Critically ill pediatric patients who require central catheterization are especially fragile, making prediction of the optimal insertion length imperative, even for guidewire insertion [22,23]. The wire should not be too deep, which may cause endocardial injury or arrhythmia, or too shallow, which may result in sub-optimal insertion of the catheter into the superior vena cava. Special caution is needed when inserting a straight-tip rather than a j-tip wire [24].

Whether deep or not, the actual intended length of catheter insertion should be based on accurate prediction. The suggested target point of the catheter tip in this study, the carina, can be considered a safe target. The average distance between the carina and the junction of the superior vena cava and right atrium in infants and children undergoing heart surgery has been reported to be $1.5 \mathrm{~cm}$ (95\% CI 1.3-1.8 $\mathrm{cm})$ [10], with this range $(1.3$ to $1.8 \mathrm{~cm})$ constituting the clinically acceptable positive limit of error (i.e., estimate - determined value $>0$ ). Based on this consideration accurate than

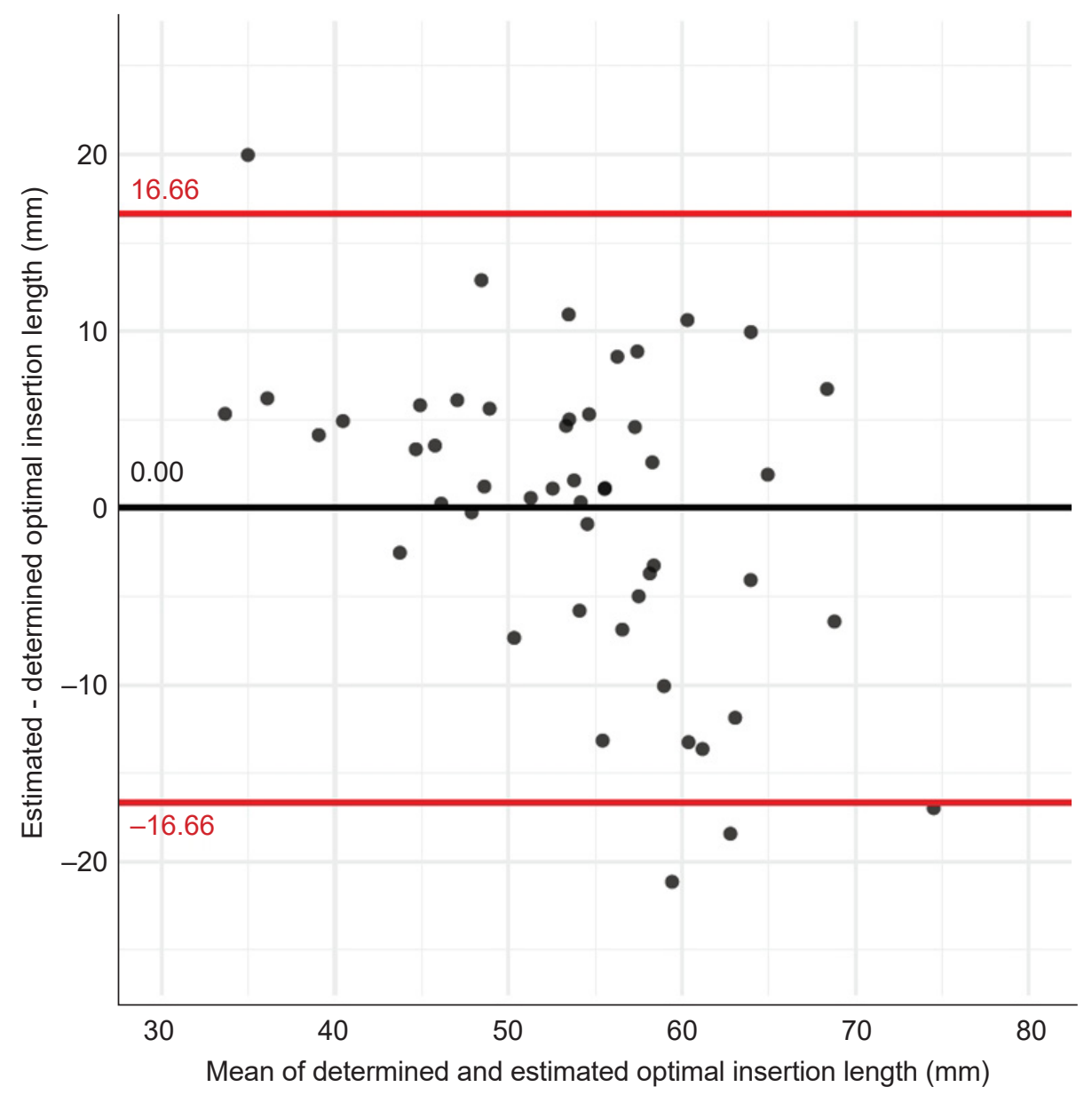

Fig. 5. Bland-Altman plot of determined and estimated optimal insertion lengths (linear regression model). 
the landmark-based method. Nevertheless, however, we concluded that the landmark-based method is also feasible in clinical practice, as the mean difference between the two estimates was -1.038 ( $95 \% \mathrm{CI}-2.4$ to $0.4 \mathrm{~mm}$ ), making them clinically identical. Also, the estimation based on the regression model requires complicated calculations, which are difficult to be performed intraoperatively. In contrast, the landmark-based method can simply estimate optimal insertion length using intraoperatively measured variables.

This study had several limitations. First, the data used in this study were not purposefully collected. Therefore, information regarding detailed complications and accompanying congenital anomalies was not recorded. Second, several patients required repeated catheterizations. Despite the time gap between procedures, autocorrelation cannot be ruled out. Third, this was a single-center study, with all procedures performed by a single clinician. Our suggested method requires external validation. Fourth, based on the limits of agreement between the estimates and the determined optimal lengths of insertion, the estimation should be regarded as a guide rather than an absolute target.

In conclusion, this study suggests that a simple landmark-based method ( $\mathrm{IA}+\mathrm{AB}-5)$ can estimate the optimal insertion length of the right subclavian vein catheterization in pediatric patients of corrected age $<1$ year.

\section{ACKNOWLEDGEMENTS}

This work was supported by Chungnam National University Hospital Research Fund.

\section{CONFLICTS OF INTEREST}

Chaeseong Lim has been an editor of the Anesthesia and Pain Medicine since 2020; however, he was not involved in the peer reviewer selection, evaluation, or decision process of this article. No other potential conflicts of interest relevant to this article were reported.

\section{DATA AVAILABILITY STATEMENT}

The datasets generated during and/or analyzed during the current study are available from the corresponding author on reasonable request.

\section{AUTHOR CONTRIBUTIONS}

Conceptualization: Boohwi Hong, Woosuk Chung, YoonHee Kim. Data curation: Hoseop Kim. Formal analysis: Chahyun Oh. Funding acquisition: Sun Yeul Lee. Methodology: Boohwi Hong, Chaeseong Lim. Project administration: Ah Young Choi, Chaeseong Lim. Visualization: Chahyun Oh. Writing - original draft: Chahyun Oh. Writing - review \& editing: Boohwi Hong, Yumin Jo, Suyeon Shin, Youngkwon Ko, Yoon-Hee Kim. Investigation: Boohwi Hong, Yumin Jo, Woosuk Chung, Hoseop Kim, Suyeon Shin, Ah Young Choi. Resources: Ah Young Choi, Sun Yeul Lee. Supervision: Youngkwon Ko, Sun Yeul Lee.

\section{ORCID}

Chahyun Oh, https://orcid.org/0000-0001-8344-4245

Boohwi Hong, https://orcid.org/0000-0003-2468-9271

Yumin Jo, https://orcid.org/0000-0002-4847-0250

Woosuk Chung, https://orcid.org/0000-0002-6409-2325

Hoseop Kim, https://orcid.org/0000-0001-6414-7050

Suyeon Shin, https://orcid.org/0000-0003-3683-7139

Ah Young Choi, https://orcid.org/0000-0002-9634-3950

Chaeseong Lim, https://orcid.org/0000-0002-2356-8999

Youngkwon Ko, https://orcid.org/0000-0002-0178-6346

Yoon-Hee Kim, https://orcid.org/0000-0002-8282-610X

Sun Yeul Lee, https://orcid.org/0000-0002-6124-5138

\section{REFERENCES}

1. Ares G, Hunter CJ. Central venous access in children: indications, devices, and risks. Curr Opin Pediatr 2017; 29: 340-6.

2. Citak A, Karaböcüoğlu M, Uçsel R, Uzel N. Central venous catheters in pediatric patients - subclavian venous approach as the first choice. Pediatr Int 2002; 44: 83-6.

3. Brass P, Hellmich M, Kolodziej L, Schick G, Smith AF. Ultrasound guidance versus anatomical landmarks for subclavian or femoral vein catheterization. Cochrane Database Syst Rev 2015; 1: CD011447.

4. Pirotte T, Veyckemans F. Ultrasound-guided subclavian vein cannulation in infants and children: a novel approach. Br J Anaesth 2007; 98: 509-14.

5. Park SI, Kim YH, So SY, Kim MJ, Kim HJ, Kim JK. Ultrasound-guided subclavian catheterization in pediatric patients with a linear probe: a case series. Korean J Anesthesiol 2013; 64: 541-4.

6. Kim KO, Jo JO, Kim HS, Kim CS. Positioning internal jugular venous catheters using the right third intercostal space in children. Acta Anaesthesiol Scand 2003; 47: 1284-6. 
7. Andropoulos DB, Bent ST, Skjonsby B, Stayer SA. The optimal length of insertion of central venous catheters for pediatric patients. Anesth Analg 2001; 93: 883-6.

8. Na HS, Kim JT, Kim HS, Bahk JH, Kim CS, Kim SD. Practical anatomic landmarks for determining the insertion depth of central venous catheter in paediatric patients. Br J Anaesth 2009; 102: 820-3.

9. Choi YJ, Hahm KD, Kwon K, Lee EH, Ro YJ, Yang HS. [Bedside prediction of right subclavian venous catheter insertion length]. Rev Bras Anestesiol 2014; 64: 419-24. Portuguese.

10. Yoon SZ, Shin JH, Hahn S, Oh AY, Kim HS, Kim SD, et al. Usefulness of the carina as a radiographic landmark for central venous catheter placement in paediatric patients. $\mathrm{Br}$ J Anaesth 2005; 95: 514-7.

11. Engle WA; American Academy of Pediatrics Committee on Fetus and Newborn. Age terminology during the perinatal period. Pediatrics 2004; 114: 1362-4.

12. Giavarina D. Understanding Bland Altman analysis. Biochem Med (Zagreb) 2015; 25: 141-51.

13. Kang SS, Shin YS, Lee SY, Kim H. Simplified equation for determining proper depth of peripherally inserted central catheter in relation to anatomical landmarks. Korean J Anesthesiol 2018; 71: 300-4.

14. Yoon SZ, Shin TJ, Kim HS, Lee J, Kim CS, Kim SD, et al. Depth of a central venous catheter tip: length of insertion guideline for pediatric patients. Acta Anaesthesiol Scand 2006; 50: 355-7.

15. Kim H, Jeong CH, Byon HJ, Shin HK, Yun TJ, Lee JH, et al. Predicting the optimal depth of left-sided central venous catheters in children. Anaesthesia 2013; 68: 1033-7.

16. Bannon MP, Heller SF, Rivera M. Anatomic considerations for central venous cannulation. Risk Manag Healthc Policy 2011; 4: 27-39.

17. Vesely TM. Central venous catheter tip position: a continuing controversy. J Vasc Interv Radiol 2003; 14: 527-34.

18. III. NKF-K/DOQI clinical practice guidelines for vascular access: update 2000. Am J Kidney Dis 2001; 37(1 Suppl 1): S137-8.

19. Booth SA, Norton B, Mulvey DA. Central venous catheterization and fatal cardiac tamponade. Br J Anaesth 2001; 87: 298302.

20. Sasaki TM, Panke TW, Dorethy JF, Lindberg RB, Pruitt BA. The relationship of central venous and pulmonary artery catheter position to acute right-sided endocarditis in severe thermal injury. J Trauma 1979; 19: 740-3.

21. Huang YC, Huang JC, Chen SC, Chang JM, Chen HC. Lethal cardiac arrhythmia during central venous catheterization in a uremic patient: a case report and review of the literature. Hemodial Int 2013; 17: 644-8.

22. Dwivedi S, Siddiqui F, Patel M, Cardozo S. Guide wire induced cardiac tamponade: the soft J tip is not so benign. Case Rep Crit Care 2016; 2016: 1436924.

23. Cavatorta F, Campisi S, Fiorini F. Fatal pericardial tamponade by a guide wire during jugular catheter insertion. Nephron 1998; 79: 352.

24. Dube SK, Chaturvedi A. A simple technique to avoid difficulty in guide wire insertion during pediatric central venous cannulation. Saudi J Anaesth 2014; 8: 141-2. 